\title{
Uptake of folic acid supplements before and during pregnancy: a study of women's views and experiences
}

\author{
A. S. Anderson ${ }^{1}$, R. Barbour ${ }^{2}$, M. Macleod ${ }^{1,3}$ and G. Mires ${ }^{3}$ \\ ${ }^{1}$ Centre for Public Health Nutrition Research, ${ }^{2}$ School of Nursing and Midwifery and ${ }^{3}$ Centre for Academic Clinical \\ Practice, College of Medicine, Dentistry and Nursing, University of Dundee, Dundee DD1 9SY, UK
}

To help reduce the risk of neural tube defects (NTD), current recommendations are that all women who could become pregnant should take a $400 \mu \mathrm{g}$ folic acid supplement per day prior to conception and continue to do so until the 12th week of pregnancy ${ }^{(1)}$. While it is recognised that compliance with this guidance is sub-optimal ${ }^{(2)}$, little is known about the reasons why.

Women attending baby clinics and existing community groups in Fife, Scotland who had a healthy baby up to 20 weeks old were approached to provide information on their use of folic acid supplements before and during their latest pregnancy. A purposive sample to reflect a range of ages, parities, socio-economic and educational levels were invited to participate in focus group discussions to explore their beliefs, understanding and rationale surrounding supplement use.

Two hundred and ninety two women were approached regarding participation and $211(70 \%)$ provided information on their folic acid supplement use. Of these, $26(12 \%)$ had not taken it at all, 67 (32\%) reported starting supplements pre-conceptually and $118(56 \%)$ only during pregnancy. However, $22(18 \%)$ of women reporting supplement use in pregnancy stated that this was taken intermittently. Multiparous women were significantly more likely $(P<0.05)$ to report not having taken the supplement at any time $(17.1 \%)$ compared to primiparous $(7.5 \%)$

A total of five focus groups and three joint interviews were undertaken with 24 women. Their ages ranged from 19 to 40 years with equal numbers of primiparous and multiparous mothers. The rationale behind the recommendation was generally known (although folic acid was also thought to relate to enhancing chances of conception, reducing miscarriage and having a positive impact on development of healthy bones and intelligence). The message was often perceived to be lost in the abundance of pregnancy communications and it was reported that it was rarely stressed by health professionals. Participants reported a perceived link between folic acid use and morning sickness, and also invoked busy lives, competing priorities and poor memory in accounting for intermittent use. Building a 'lay evidence base' from their own experiences, many cited healthy pregnancy outcomes without supplement use and expressed scepticism about its preventive action.

The results provide guidance on areas that should be incorporated into future programmes including the importance of daily folic acid supplement use, the severity of NTD and the evidence on risk reduction. The importance of the inter-partum period is also highlighted.

The funding was provided by Jennifer Brown Research Fund Fife Appeal supported by PiggyBankKids and administered by NHS Fife. Recruitment was greatly assisted by Pamela Galloway, midwife, NHS Fife.

1. Scientific Advisory Committee on Nutrition (SACN) (2007) Folate and Disease Prevention SACN. London: TSO

2. Ray JG, Singh G \& Burrows RF (2004) Evidence for suboptimal use of periconceptional folic acid supplements globally Br J Obstet Gynaecol. 111, 339-408. 